\title{
LYAPUNOV-TYPE INEQUALITIES FOR A CLASS THIRD-ORDER DIFFERENTIAL EQUATIONS
}

\author{
YONG FANG QI ${ }^{1}$, YOUHUA PENG ${ }^{1}$ \\ and XUHUAN WANG ${ }^{1,2}$ \\ ${ }^{1}$ Department of Mathematics \\ Pingxiang University \\ Pingxiang 337000 \\ Jiangxi \\ P. R. China \\ e-mail: wangxuhuan84@163.com \\ ${ }^{2}$ Department of Education Science \\ Pingxiang University \\ Pingxiang, 337000 \\ Jiangxi \\ P. R. China
}

\begin{abstract}
In this paper, we present Lyapunov-type inequalities for a class third-order differential equations, the result is novel and generalizes some early results on this topic. We obtained an interval where the equations have no nontrivial solutions.
\end{abstract}

\section{Introduction}

The well-known Lyapunov inequality [1] states that if $x(t)$ is a solution of

2010 Mathematics Subject Classification: 26A33, 34B15.

Keywords and phrases: Lyapunov's inequality, Euler equation, boundary conditions, Grönwall's inequality, nontrivial solutions.

Received May 31, 2017

(C) 2017 Scientific Advances Publishers 


$$
x^{\prime \prime}(t)+p(t) x(t)=0,
$$

where $t \in[a, b]$ and with the condition $x(a)=x(b)=0$, then the inequality

$$
\int_{a}^{b}|p(t)| d t>\frac{4}{b-a}
$$

holds. This result plays an important role in the study of various problems such as oscillation, disconjugacy, eigenvalue problems, and many other problems of differential equations. Due to its importance, the Lyapunov inequality has been improved and generalized in many forms. Nehari ([2], Theorem I) claims that if an interval $[a, b]$ contains $n$ zeros of a nontrivial solution of

$$
x^{(n)}(t)+p_{n} x^{(n-1)}(t)+\cdots+p_{1} x(t)=0 .
$$

Then

$$
\sum_{k=1}^{n} 2^{k}(b-a)^{n-k} \int_{a}^{b}|p(t)| d t>2^{n+1} .
$$

But in a private communication with one of the authors, Nehari has indicated that the inequality is undecided and the above result is incorrect [3]. In [3], Fink and St. Mary gave the Lyapunov-type inequalities for the following equation:

$$
x^{\prime \prime}(t)+g(t) x^{\prime}(t)+f(t) x(t)=0
$$

The result is

$$
(b-a) \int_{a}^{b} f^{+}(t) d t+2 \int_{a}^{b}|g(t)| d t>4 .
$$

It is well-known that Lyapunov-type inequalities have been extended to higher order linear differential equations. Parhi and Panigrahi [4] established the following results for the third-order linear equation:

$$
x^{\prime \prime \prime}(t)+p(t) x(t)=0 .
$$


Assume Equation (7) has a solution $x(t)$, such that $x(a)=x(b)=0$ and $x(t) \neq 0$ for $t \in(a, b)$, suppose there exists $d \in(a, b)$ such that $x^{\prime \prime}(d)=0$, then

$$
\int_{a}^{b}|p(t)| d t>\frac{4}{(b-a)^{2}}
$$

Assume Equation (7) has a solution $x(t)$, such that $x(a)=x(b)=x(c)=0$ and $x(t) \neq 0$ for $t \in(a, b) \cup(b, c)$, then

$$
\int_{a}^{c}|p(t)| d t>\frac{4}{(c-a)^{2}}
$$

Yang [5] generalized the results given above for third-order linear equations to the $n$-th order linear equation

$$
x^{(n)}(t)+p(t) x(t)=0
$$

where $p(t) \in C([a, b], R)$, for both cases when $n$ is even and odd. His work was further improved by Çakmak [6].

The Lyapunov inequality has proved to be very useful in various problems related with differential equations [7, 8, 9], many of its generalizations have proved to be useful tools in oscillation theory, eigenvalue problems ([10], Corollaries 2.1 and 2.2) and numerous other applications for the theories of differential equations [11].

Motivated by the above works, we investigate the third-order differential equations by using the integral method and Grönwall's inequality. More precisely, we are interested with the following differential equations:

$$
x^{\prime \prime \prime}(t)+p(t) x^{\prime \prime}(t)+x^{\prime}(t)+x(t)=0,
$$

where $t \in[a, b], d \in(a, b), x(a)=x(d)=x(b)=0$. And

$$
\left(\left(r_{2}(t)\left(r_{1}(t) x^{\prime}\right)^{\prime}\right)^{\prime}+x^{\prime \prime}+x^{\prime}+q(t) x=0 .\right.
$$




\section{Preliminaries}

In this section, we introduce technical lemma that will be crucial in establishing the main result.

Lemma 1 (Grönwall's inequality). Let $K>0, f(t), g(t) \geq 0$ and $f(t)$, $g(t)$ are continuous functions. If the following inequality holds:

$$
f(t) \leq K+\int_{t}^{b} f(s) g(s) d s, \quad t \in[a, b]
$$

then

$$
f(t) \leq K e^{\int_{a}^{b} g(s) d s}, \quad t \in[a, b]
$$

Proof. The usual proof is as follows. Considering the conditions given above, we have

$$
\frac{f(t)}{K+\int_{t}^{b} f(s) g(s) d s} \leq 1 .
$$

Multiply this by $g(t)$ to get

$$
\left|\frac{d}{d t} \ln \left(K+\int_{t}^{b} f(s) g(s) d s\right)\right| \leq g(t),
$$

implies that

$$
-g(t) \leq \frac{d}{d t} \ln \left(K+\int_{t}^{b} f(s) g(s) d s\right) \leq g(t)
$$

It is obvious that

$$
-\int_{t}^{b} g(t) d t \leq \int_{t}^{b} 1 d \ln \left(K+\int_{t}^{b} f(s) g(s) d s\right) \leq \int_{t}^{b} g(t) d t
$$

therefore, 


$$
\left|\int_{t}^{b} 1 d \ln \left(K+\int_{t}^{b} f(s) g(s) d s\right)\right| \leq \int_{t}^{b} g(t) d t .
$$

Then we have

$$
\left|\ln \left(K+\int_{t}^{b} f(s) g(s) d s\right)-\ln K\right|=\ln \frac{K+\int_{t}^{b} f(s) g(s) d s}{K} \leq \int_{t}^{b} g(t) d t
$$

that is,

$$
K+\int_{t}^{b} f(s) g(s) d s \leq K e^{\int_{t}^{b} g(t) d t}
$$

By (12), we obtain

$$
f(t) \leq K+\int_{t}^{b} f(s) g(s) d s \leq K e^{\int_{t}^{b} g(t) d t} \leq K e^{\int_{a}^{b} g(t) d t},
$$

which implies that $f(t) \leq K e^{\int_{a}^{b} g(s) d s}$, the proof is completed.

\section{Main Results}

Theorem 1. Considers the following differential equation:

$$
x^{\prime \prime \prime}(t)+p(t) x^{\prime \prime}(t)+x^{\prime}(t)+x(t)=0,
$$

where $p(t)$ is integrable on $[a, b], t \in[a, b]$ and $d \in(a, b)$. Suppose $x(t)$ is a nontrivial solution of (22), and $a, b, d$ are successive zeros satisfying $x(a)=x(d)=x(b)=0$. Then the following inequality is obtained:

$$
\int_{a}^{b}|p(t)| d t \geq-\ln \left[\left(\frac{b-a}{2}\right)^{4}\left(1+\frac{b-a}{2}\right)^{2}\right] .
$$

Proof. Let

$$
\begin{aligned}
& x_{1}=x^{\prime}, \\
& x_{2}=x_{1}^{\prime},
\end{aligned}
$$




$$
x_{2}^{\prime}+p(t) x_{2}+x_{1}^{\prime}+x=0 \text {. }
$$

Since $x(t)$ vanishes three times on $[a, b]$, each $x_{i}(i=1,2)$ vanishes at least once on that interval. Therefore, we can reasonably conclude that there exist $d_{1} \in(a, d), d_{2} \in(d, b), c \in\left(d_{1}, d_{2}\right)$, such that $x^{\prime}\left(d_{1}\right)=x^{\prime}\left(d_{2}\right)=0$, $x^{\prime \prime}(c)=0$, where $d_{1}<d_{2}$. We split $[a, b]$ into two subintervals $[a, c]$ and $[c, b]$ such that on each, $x_{1}$ vanishes at least once. Since $x_{1}=x^{\prime}$, integrating this equation from $a$ to $t \in(a, c), \int_{a}^{t} x_{1} d t=\int_{a}^{t} x^{\prime} d t$. Considering $x(a)=0$, we have

$$
|x(t)| \leq \int_{a}^{t}\left|x_{1}\right| d t \leq \int_{a}^{c}\left|x_{1}\right| d t \leq\left|x_{1}(t)\right|_{\max }(c-a)
$$

where $\left|x_{1}(t)\right|_{\max }=\max \left(\left|x_{1}(t)\right|\right), t \in[a, c]$.

Since $x^{\prime}\left(d_{1}\right)=0$ and $x_{1}=x^{\prime}$ imply that $x_{1}\left(d_{1}\right)=0$. By $x_{2}=x_{1}^{\prime}$, integrating this equation from $d_{1}$ to $t \in\left(d_{1}, c\right)$. It is easy to get $x_{1}(t)=\int_{d_{1}}^{t} x_{2} d t$ and

$$
\left|x_{1}(t)\right| \leq \int_{d_{1}}^{t}\left|x_{2}\right| d t \leq \int_{d_{1}}^{c}\left|x_{2}\right| d t \leq\left|x_{2}(t)\right|_{\max }\left(c-d_{1}\right) \leq\left|x_{2}(t)\right|_{\max }(c-a),
$$

where $\left|x_{2}(t)\right|_{\max }=\max \left(\left|x_{2}(t)\right|\right), \mathbf{t} \in[\mathbf{a}, \mathbf{c}]$.

Notice that $\left|x_{2}(t)\right|_{\max }=\max \left(\left|x_{2}(t)\right|\right), \mathbf{t} \in\left[\mathbf{d}_{1}, \mathbf{c}\right]$, (28) can not be plugged into (31).

With $x^{\prime \prime}(c)=0$, this implies that $x_{2}(c)=0$. Integrating (26) from $c$ to $t \in(a, c)$, it is easy to get

$$
\int_{c}^{t} x_{2}^{\prime} d t+\int_{c}^{t} p(t) x_{2} d t+\int_{c}^{t} x_{1} d t+\int_{c}^{t} x d t=0
$$

Clearly, 


$$
x_{2}(t)+\int_{c}^{t} p(t) x_{2} d t+\int_{c}^{t} x_{1} d t+\int_{c}^{t} x d t=0,
$$

implies that

$$
\begin{array}{r}
x_{2}(t)=-\int_{c}^{t} p(t) x_{2} d t-\int_{c}^{t} x_{1} d t-\int_{c}^{t} x d t, \\
x_{2}(t)=\int_{t}^{c} p(t) x_{2} d t+\int_{t}^{c} x_{1} d t+\int_{t}^{c} x d t, \\
\left|x_{2}\right| \leq \int_{t}^{c}|p(t)|\left|x_{2}\right| d t+\int_{a}^{c}\left|x_{1}\right| d t+\int_{a}^{c}|x| d t .
\end{array}
$$

Substituting (27) and (28) into (31), we arrive at

$$
\left|x_{2}\right| \leq\left|x_{2}\right|_{\max }(c-a)^{2}\left|x_{2}\right|_{\max }(c-a)^{3}+\int_{t}^{c}|p(t)|\left|x_{2}\right| d t,
$$

with the lemma (Grönwall's inequality) given above, the following inequality is obtained:

$$
\left|x_{2}\right| \leq\left[\left|x_{2}\right|_{\max }(c-a)^{2}+\left|x_{2}\right|_{\max }(c-a)^{3}\right] e^{\int_{a}^{c}|p(t)| d t},
$$

the inequality above holds for every $t \in[a, c]$, so

$$
\left|x_{2}\right|_{\max } \leq\left[\left|x_{2}\right|_{\max }(c-a)^{2}+\left|x_{2}\right|_{\max }(c-a)^{3}\right] e^{\int_{a}^{c}|p(t)| d t},
$$

we get

$$
1 \leq\left[(c-a)^{2}+(c-a)^{3}\right] e^{\int_{a}^{c}|p(t)| d t} .
$$

Therefore,

$$
\int_{a}^{c}|p(t)| d t \geq \ln \frac{1}{(c-a)^{2}+(c-a)^{3}} .
$$


Similarly,

$$
\int_{c}^{b}|p(t)| d t \geq \ln \frac{1}{(b-c)^{2}+(b-c)^{3}}
$$

From (36) and (37), we obtain

$$
\begin{aligned}
\int_{a}^{b}|p(t)| d t & =\ln \frac{1}{(c-a)^{2}+(c-a)^{3}}+\ln \frac{1}{(b-c)^{2}+(b-c)^{3}} \\
& =-\ln \left\{\left[(c-a)^{2}+(c-a)^{3}\right]\left[(b-c)^{2}+(b-c)^{3}\right]\right\}
\end{aligned}
$$

Let

$$
\begin{aligned}
g(c) & =\left[(c-a)^{2}+(c-a)^{3}\right]\left[(b-c)^{2}+(b-c)^{3}\right] \\
& =(c-a)^{2}(b-c)^{2}[1+(c-a)][1+(b-c)] \\
& =\left[-\left(c-\frac{a+b}{2}\right)^{2}+\frac{(a-b)^{2}}{4}\right]^{2}\left[-\left(c-\frac{a+b}{2}\right)^{2}+\left(\frac{b-a}{2}+1\right)^{2}\right] .
\end{aligned}
$$

When $c \in(a, b)$, it is clearly that $0<-\left(c-\frac{a+b}{2}\right)^{2}+\frac{(a-b)^{2}}{4} \leq \frac{(a-b)^{2}}{4}$,

$0<-\left(c-\frac{a+b}{2}\right)^{2}+\left(\frac{b-a}{2}+1\right)^{2} \leq\left(\frac{b-a}{2}+1\right)^{2}$.

Thus,

$$
0<g(c) \leq\left(\frac{a-b}{2}\right)^{4}\left(\frac{b-a}{2}+1\right)^{2}, \quad c \in(a, b)
$$

when $c=\frac{a+b}{2}$,

$$
g(c)=\left(\frac{a-b}{2}\right)^{4}\left(\frac{b-a}{2}+1\right)^{2}
$$

considering (38), we have 


$$
\int_{a}^{b}|p(t)| d t \geq-\ln \left[\left(\frac{b-a}{2}\right)^{4}\left(1+\frac{b-a}{2}\right)^{2}\right] .
$$

This completes the proof of Theorem 1 .

Application. Considering the following Euler equation:

$$
x^{3} y^{\prime \prime \prime}(x)+x^{2} y^{\prime \prime}(x)+y(x)=0
$$

where $b>a>0$, suppose $y$ is a nontrivial solution of the system above, and satisfying $y(\ln a)=y(\ln d)=y(\ln b)=0$, for $d \in(a, b)$. Then

$$
\frac{b}{a}\left(\ln \frac{b}{a}\right)^{2}\left(2+\ln \frac{b}{a}\right) \geq 8 .
$$

Proof. Let $x=e^{t}, t=\ln x, x \in[a, b], a>0, b>0$ and $t \in[\ln a$, $\ln b]$.

$$
\begin{gathered}
\frac{d y}{d x}=\frac{d y}{d t} \frac{d t}{d x}=e^{-t} \frac{d y}{d t}, \\
\frac{d^{2} y}{d x^{2}}=e^{-2 t}\left(\frac{d^{2} y}{d t^{2}}-\frac{d y}{d t}\right), \\
\frac{d^{3} y}{d x^{3}}=e^{-3 t}\left(\frac{d^{3} y}{d t^{3}}-3 \frac{d^{2} y}{d t^{2}}+2 \frac{d y}{d t}\right) .
\end{gathered}
$$

We can transform (39) to the following equation:

$$
y^{\prime \prime \prime}(t)-2 y^{\prime \prime}(t)+y^{\prime}(t)+y(t)=0 .
$$

With the result of Theorem 1, we obtain

$$
\begin{gathered}
\int_{\ln a}^{\ln b}|-2| d t \geq-\ln \left[\left(\frac{\ln b-\ln a}{2}\right)^{4}\left(1+\frac{\ln b-\ln a}{2}\right)^{2}\right] \\
2(\ln b-\ln a) \geq-\ln \left[\left(\frac{\ln b-\ln a}{2}\right)^{4}\left(1+\frac{\ln b-\ln a}{2}\right)^{2}\right], \\
2 \ln \frac{b}{a} \geq \ln \left[\left(\frac{\ln b-\ln a}{2}\right)^{4}\left(1+\frac{\ln b-\ln a}{2}\right)^{2}\right]^{-1}
\end{gathered}
$$




$$
\begin{aligned}
\ln \left(\frac{b}{a}\right)^{2} & \geq \ln \left[\left(\frac{2}{\ln b-\ln a}\right)^{4}\left(\frac{2}{2+\ln b-\ln a}\right)^{2}\right], \\
\left(\frac{b}{a}\right)^{2} & \geq\left(\frac{2}{\ln b-\ln a}\right)^{4}\left(\frac{2}{2+\ln b-\ln a}\right)^{2},
\end{aligned}
$$

since $b>a>0$, then

$$
\begin{gathered}
\frac{b}{a} \geq \frac{8}{(\ln b-\ln a)^{2}(2+\ln b-\ln a)}=\frac{8}{\left(\ln \frac{b}{a}\right)^{2}\left(2+\ln \frac{b}{a}\right)}, \\
\frac{b}{a}\left(\ln \frac{b}{a}\right)^{2}\left(2+\ln \frac{b}{a}\right) \geq 8 .
\end{gathered}
$$

This completes the proof.

Theorem 2. Considers the following third-order half-linear equation:

$$
\left(\left(r_{2}(t)\left(r_{1}(t) x^{\prime}\right)^{\prime}\right)^{\prime}+x^{\prime \prime}+x^{\prime}+q(t) x=0,\right.
$$

where $r_{k} \in C^{2-k+1}\left([a, b], R^{+}\right), k=1,2, q(t) \in C([a, b], R)$, if $x(t)$ is a nonzero solution of (43) satisfying $x(a)=x(b)=0$ and there exists $d \in(a, b)$ such that $f_{1}(d)=0$, where $f_{1}(t)=\left(r_{1}(t) x^{\prime}\right)^{\prime}, k=\min \left\{r_{1}(t)\right\}, t \in[a, b]$, then

$$
\int_{a}^{b}|q(t)| d t \geq \frac{1}{\int_{a}^{b} \frac{1}{r_{1}(t)} d t \int_{a}^{b} \frac{1}{r_{2}(t)} d t} \ln \frac{1}{\frac{2 \int_{a}^{b} \frac{1}{r_{2}(t)} d t}{k}+\int_{a}^{b} \frac{1}{r_{2}(t)} d t \int_{a}^{b} \frac{1}{r_{1}(t)} d t}
$$

Proof. In order to prove the theorem, we define functions $x_{k}$, $k=1,2$, as follows:

$$
\begin{gathered}
x^{\prime}=\frac{x_{1}}{r_{1}(t)}, \\
x_{1}^{\prime}=\frac{x_{2}}{r_{2}(t)},
\end{gathered}
$$




$$
x_{2}^{\prime}=-\left[x^{\prime \prime}+\frac{x_{1}}{r_{1}(t)}+q(t) x\right] \text {. }
$$

Since $x^{\prime}=\frac{x_{1}}{r_{1}(t)}, x(a)=0$ and $t \in[a, b]$, we have

$$
\int_{a}^{t} x^{\prime}(t) d t=\int_{a}^{t} \frac{x_{1}}{r_{1}(t)} d t
$$

Therefore,

$$
|x(t)| \leq \int_{a}^{b} \frac{\left|x_{1}\right|}{r_{1}(t)} d t
$$

Since $x(a)=x(b)=0$. There exists $d_{1} \in(a, b)$ such that $x^{\prime}\left(d_{1}\right)=0$, implying $x_{1}\left(d_{1}\right)=0$, combining with (46), we have

$$
\int_{d_{1}}^{t} x_{1}^{\prime}(t) d t=\int_{d_{1}}^{t} \frac{x_{2}}{r_{2}(t)} d t
$$

Clearly,

$$
\left|x_{1}(t)\right| \leq \int_{d_{1}}^{t} \frac{\left|x_{2}\right|}{r_{2}(t)} d t \leq \int_{a}^{b} \frac{\left|x_{2}\right|}{r_{2}(t)} d t \leq\left|x_{2}\right|_{\infty} \int_{a}^{b} \frac{1}{r_{2}(t)} d t
$$

where $\left|x_{2}\right|_{\infty}=\max \left\{\left|x_{2}(t)\right|\right\}, t \in[a, b]$.

If $t \in[d, b]$, with the condition $f_{1}(d)=0$, implying $x_{2}(d)=0$, combining with (47), (49) and (51), we have

$$
\begin{aligned}
\int_{d}^{t} x_{2}^{\prime}(t) d t & =-\left[\int_{d}^{t} x^{\prime \prime} d t+\int_{d}^{t} \frac{x_{1}}{r_{1}(t)} d t+\int_{d}^{t} q(t) x(t) d t\right] \\
& =-\left[x^{\prime}(t)-x^{\prime}(d)+\int_{d}^{t} \frac{x_{1}}{r_{1}(t)} d t+\int_{d}^{t} q(t) x(t) d t\right],
\end{aligned}
$$

implies that 


$$
\begin{aligned}
\left|x_{2}(t)\right| \leq & \left|x^{\prime}(t)\right|+\left|x^{\prime}(d)\right|+\int_{a}^{b} \frac{\left|x_{1}\right|}{r_{1}(t)} d t+\int_{d}^{t}|q(t)||x(t)| d t \\
\leq & \frac{\left|x_{1}(t)\right|}{k}+\frac{\left|x_{1}(d)\right|}{k}+\int_{a}^{b} \frac{\left|x_{1}\right|}{r_{1}(t)} d t+\int_{d}^{t}|q(t)||x(t)| d t \\
\leq & \frac{\left|x_{2}\right|_{\infty} \int_{a}^{b} \frac{1}{r_{2}(t)} d t}{k}+\frac{\left|x_{2}\right|_{\infty} \int_{a}^{b} \frac{1}{r_{2}(t)} d t}{k}+\left|x_{2}\right|_{\infty} \int_{a}^{b} \frac{1}{r_{2}(t)} d t \int_{a}^{b} \frac{1}{r_{1}(t)} d t \\
& +\int_{d}^{t}|q(t)|\left|x_{2}(t)\right| \int_{a}^{b} \frac{1}{r_{1}(t)} d t \int_{a}^{b} \frac{1}{r_{2}(t)} d t d t \\
\leq & \frac{2\left|x_{2}\right|_{\infty} \int_{a}^{b} \frac{1}{r_{2}(t)} d t}{k}+\left|x_{2}\right|_{\infty} \int_{a}^{b} \frac{1}{r_{2}(t)} d t \int_{a}^{b} \frac{1}{r_{1}(t)} d t \\
& +\int_{d}^{t}|q(t)|\left|x_{2}(t)\right| \int_{a}^{b} \frac{1}{r_{1}(t)} d t \int_{a}^{b} \frac{1}{r_{2}(t)} d t
\end{aligned}
$$

where $k=\min \left\{r_{1}(t)\right\}, t \in[a, b]$. According to the classic Grönwall's inequality, it is obvious that

$$
\begin{array}{r}
\left|x_{2}(t)\right| \leq\left(\frac{2\left|x_{2}\right|_{\infty} \int_{a}^{b} \frac{1}{r_{2}(t)} d t}{k}+\left|x_{2}\right|_{\infty} \int_{a}^{b} \frac{1}{r_{2}(t)} d t \int_{a}^{b} \frac{1}{r_{1}(t)} d t\right) \\
\times e^{\int_{d}^{t}|q(t)| d t \int_{a}^{b} \frac{1}{r_{1}(t)} d t \int_{a}^{b} \frac{1}{r_{2}(t)} d t},
\end{array}
$$

that is,

$$
\left|x_{2}\right|_{\infty} \leq\left(\frac{2\left|x_{2}\right|_{\infty} \int_{a}^{b} \frac{1}{r_{2}(t)} d t}{k}+\left|x_{2}\right|_{\infty} \int_{a}^{b} \frac{1}{r_{2}(t)} d t \int_{a}^{b} \frac{1}{r_{1}(t)} d t\right)
$$




$$
\times e^{\int_{a}^{b}|q(t)| d t \int_{a}^{b} \frac{1}{r_{1}(t)} d t \int_{a}^{b} \frac{1}{r_{2}(t)} d t} .
$$

Then we have

$$
1 \leq\left[\frac{2 \int_{a}^{b} \frac{1}{r_{2}(t)} d t}{k}+\int_{a}^{b} \frac{1}{r_{2}(t)} d t \int_{a}^{b} \frac{1}{r_{1}(t)} d t\right] e^{\int_{a}^{b}|q(t)| d t \int_{a}^{b} \frac{1}{r_{1}(t)} d t \int_{a}^{b} \frac{1}{r_{2}(t)} d t} .
$$

We obtain

$$
\frac{1}{\int_{a}^{b} \frac{1}{r_{1}(t)} d t \int_{a}^{b} \frac{1}{r_{2}(t)} d t} \ln \frac{1}{\frac{2 \int_{a}^{b} \frac{1}{r_{2}(t)} d t}{k}+\int_{a}^{b} \frac{1}{r_{2}(t)} d t \int_{a}^{b} \frac{1}{r_{1}(t)} d t} \leq \int_{a}^{b}|q(t)| d t .
$$

Similarly, if $t \in[a, d]$, applying Lemma 1 (Grönwall's inequality) given above, we can get the same result. This completes the proof of Theorem 2 .

Corollary 1. Assume

$$
\int_{a}^{b}|p(t)| d t<-\ln \left[\left(\frac{b-a}{2}\right)^{4}\left(1+\frac{b-a}{2}\right)^{2}\right],
$$

then the Equation (22) together with the boundary conditions $x(a)=x(d)$ $=x(b)=0$ has no nontrivial solution, $d \in(a, b)$.

\section{Corollary 2. Assume}

$$
\int_{a}^{b}|q(t)| d t<\frac{1}{\int_{a}^{b} \frac{1}{r_{1}(t)} d t \int_{a}^{b} \frac{1}{r_{2}(t)} d t} \ln \frac{1}{\frac{2 \int_{a}^{b} \frac{1}{r_{2}(t)} d t}{k}+\int_{a}^{b} \frac{1}{r_{2}(t)} d t \int_{a}^{b} \frac{1}{r_{1}(t)} d t}
$$

then the Equation (47) together with the conditions in Theorem 2 has no nontrivial solution, where $k=\min \left\{r_{1}(t)\right\}, t \in[a, b]$. 


\section{Summary}

The purpose of this paper is to provide the inequalities for a class third-order differential equations, the result is generalizes some early results on this topic [3]. Firstly, we study the third-order differential equation by using the integral method and Grönwall's inequality. Similarly, we obtained the same results for Equation (39) and half-linear equation. Finally, we give two corollaries of nontrivial solutions of the above theorems.

\section{Acknowledgement}

This work was supported by the National Natural Science Foundation of China (Grant No. 11661065) and by Scientific Research Foundation of Jiangxi Provincial Education Department (Grant No. GJJ161265).

\section{References}

[1] A. M. Lyapunov, Problème général de la stabilité de movement, Princeton Univ. Press, Princeton, N. J., 1947.

[2] Z. Nehari, On an Inequality of Lyapunov Studies in Mathematical Analysis and Related Topics, Stanford Univ. Press, Stanford, California, 1962.

[3] A. M. Fink and D. F. St. Mary, On an inequality of Nehari, Proc. Amer. Math. Soc. 21(3) (1969), 640-642.

[4] N. Parhi and S. Panigrahi, On Lyapunov-type inequality for third-order differential equations, J. Math. Anal. Appl. 233(2) (1999), 445-460.

[5] X. Yang, On Lyapunov inequality for certain higher-order differential equations, Appl. Math. Comput. 134(2) (2003), 307-317.

[6] D. Çakmak, Lyapunov-type integral inequalities for certain higher order differential equations, Appl. Math. Comput. 216(2) (2010), 368-373.

[7] X. J. Yang, Y. InKim and K. M. Lo, Lyapunov-type inequality for a class of odd-order differential equations, J. Comput. Appl. Math. 234(10) (2010), 2962-2968.

[8] R. C. Brown and D. B. Hinton, Lyapunov inequalities and their applications, Survey on Classical Inequalities, Springer Netherlands 571 (2000), 1-25.

[9] B. G. Pachpatte, Mathematical Inequalities, North Holland Mathematical Library, Elsevier, Amsterdam, 2005. 
[10] S. Dhar and Q. K. Kong, Lyapunov-type inequality for higher order half-linear differential equations, Appl. Math. Comput. 273 (2016), 114-124.

[11] R. A. C. Ferreira, On a Lyapunov-type inequality and the zeros of a certain MittagLeffer function, J. Math. Anal. Appl. 412(2) (2014), 1058-1063. 